\title{
Cerambycidae (Coleoptera) da Colômbia. IV. Cerambycinae com olhos grosseiramente granulados
}

\author{
Ubirajara R. Martins ${ }^{1,3}$ \\ Maria Helena M. Galileo 2,3
}

\begin{abstract}
Cerambycidae (Coleoptera) from Colômbia. IV. Cerambycinae with coarsely granulated eyes. New records for Colombian Cerambycidae - Achrysonini: Achryson immaculipenne Gounelle, 1909, A. lineolatum Erichson, 1847, A. quadrimaculatum (Fabricius, 1792); Ectenessini: Ectenessa lurida Martins, 1973; Cerambycini: Jupoata rufipennnis (Gory, 1831), Poeciloxestia carlyslei Fragoso, 1978, P. paraensis Lane, 1965; Elaphidionini: Ambonus electus (Gahan \& Arrow, 1903), Paramallocera cribripennis (Bates, 1885), Periboeum pubescens (Olivier, 1790), Stizocera plicicollis (Germar, 1824), S. poeyi (Guérin-Méneville, 1838), Pantonyssus glabricollis Fuchs, 1961; Piezocerini: Haruspex lineolatus Bates, 1870; Eligmodermini: Eligmoderma spinicolle Aurivillius, 1923, E. ziczac Nonfried, 1895. New species described: Pantonyssus suturale sp. nov. (Santander); Anelaphus colombianus sp. nov. (Santander); A. sparsus sp. nov. (Magdalena).
\end{abstract}

KEYwords. Cerambycinae; Coleoptera; Colômbia; new records; new species.

\section{INTRODUÇÃO}

Baseados em material procedente da Colômbia, enviado por Claudia Martínez e Fernando Fernandez (IAHC), estudamos, nesta quarta contribuição, os Cerambycinae com olhos grosseiramente granulados, das tribos Achrysonini, Ectenessini, Cerambycini, Elaphidionini,Piezocerini e Eligmodermini. Trabalhos precedentes sobre o mesmo tema foram publicados por MarTinEZ (2000), MARTINS \& GALILEO (2002 a, b) e GALILEO \& MARTINS (2003). Objetivamos assinalar novos registros e descrever três espécies novas.

Referêncas bibliográficas restringem-se às descrições originais, ao catálogo de MonNÉ (1993a, b, c, d) e aos trabalhos posteriores a esse catálogo.

$\mathrm{O}$ material mencionado pertence às instituições: IAHC, Instituto de Investigaciones de Recursos Biológicos "Alexander van Humboldt", Villa de Leyva; GMIC, Coleção Gilberto Mendoza, Santafé de Bogotá; MCNZ, Museu de Ciências Naturais, Fundação Zoobotânica do Rio Grande do Sul, Porto Alegre; MZSP, Museu de Zoologia, Universidade de São Paulo, São Paulo.

Achrysonini

Achryson immaculipenne Gounelle, 1909

Achryson immaculipenne Gounelle, 1909b: 599; Monné, 1993a: 36 (cat.); Joly, 2000: 159, figs. 11, 17; Martins, 2002: 91, figs. 85, 86. Achryson unicolor Gounelle, 1909a: 305 non Bruch, 1908: 203.
Dados de distribuição desta espécie foram apresentados por MonNÉ (1993a) que arrolou os estados brasileiros de Mato Grosso, Goiás e Pernambuco. Joly (2000) assinalou-a para diversos estados da Venezuela. Martins (2002) ampliou consideravelmente a distribuição para Colômbia (Bolívar), Equador (Napo-Pastaza), Bolívia (Santa Cruz) e Brasil (Mato Grosso ao Paraná).

Material examinado. COLÔMBIA. Cundinamarca: Mesitas del Colegio (Vereda Martínez, 4³5'14"N, 74²6’58"W, 983 m), macho, 5.IX.1978, A. Bautista \& A. Bohórquez col. (IAHC).

\section{Achryson lineolatum Erichson, 1847}

(Fig. 1)

Achryson lineolatum Erichson, 1847:1 42; Monné, 1993a: 36 (cat.); Barriga et al., 1993: 69 (hosp.); Joly, 2000: 166, figs 13, 19; Martins, 2002: 127, figs. 159-166.

Achryson scutellatum Kirsch, 1889: 37.

Achryson philippii Germain, 1898: 560.

Achryson galapagoensis Linell, 1898: 259; Martins, 1976b: 74.

Achryson galapagoensis darwini Linsley \& Chemsak, 1966: 213, figs. $15,16$.

Achryson galapagoensis flavescens Linsley \& Chemsak, 1966: 213.

Achryson lineolatum var. andinum Tippmann, 1960: 108, est. 4, fig. 7 a.

Achryson lineolatum é variável na coloração da face ventral que, nos exemplares do sul da distribuição (Peru ao Chile), é amarelada. Exemplares citados para a Venezuela (Joly 2000) 
têm a face ventral escurecida, exceto o mesosterno e o centro do metasterno. Espécimens colombianos (Fig. 1) também apresentam essa coloração na face ventral.

A distribuição de A. lineolatum estende-se pela Venezuela, Colômbia, Equador (inclusive Arquipélago de Galápagos), Peru e Bolívia.

Material examinado. COLÔMBIA. Santander: Macaravita (Hacienda El Cairo), fêmea, XII.1992, Corradine col. (GMIC); macho, XII.1993, Corradine col. (MZSP).

Achryson quadrimaculatum (Fabricius, 1792)

Callidium 4maculatum Fabricius, 1792: 328.

Callidium quadrimaculatum; Schoenherr, 1817: 454

Achryson quadrimaculatum; Martins, 1971: 165; Monné, 1993a: 37

(cat.); Di Iorio, 1994: 19 (hosp.); 1997: 153 (hosp.); Joly, 2000:

170, figs. 14, 20.

Achryson ornatipenne Perroud, 1855: 383; Villiers, 1980: 272.

Achryson hirsutulum Bates, 1870: 248; Martins, 1976b: 73.

Stromatium kartaboensis Fisher, 1944: 1; Martins, 1976b: 73.

Achryson griseopubescens Monné, 1972: 109, fig. 17; Martins, 1976b: 73

Martins (2002) resumiu as ocorrências conhecidas para $A$. quadrimaculatum: Guatemala, El Salvador, Guadeloupe, Aruba, Trinidad y Tobago, Colômbia, Venezuela, Guiana, Guiana Francesa, Brasil (Amazonas, Pará, Mato Grosso do Sul), Bolívia e Argentina (Córdoba, Salta, Chaco, Santa Fe). As localidades colombianas mencionadas por MARTINS (1976b) foram Río Magdalena e Cundinamarca: Fusagasugá.

Material examinado. COLÔMBIA. Santander: Bochalema (7³6’53”N, 72³9’02"W, $1051 \mathrm{~m})$, macho, 14.X.1974, A. Figuera col. (IAHC).

Ectenessini

Ectenessa lurida Martins, 1973

Ectenessa lurida Martins, 1973: 203; 1998: 138; Monné, 1993a: 45 (cat.); Martínez, 2000: 85 (distr.).

Originalmente descrita de Trinidad y Tobago, Ectenessa lurida foi assinalada para Venezuela por NAPP \& MARTINS (1982) e por MARTínez (2000) para Colômbia: Chocó.

Material examinado. COLÔMBIA. Magdalena: PNN (Parque Nacional Natural) Tayrona (Pueblito, $11^{\circ} 20^{\prime} \mathrm{N}, 74^{\circ} 02^{\prime} \mathrm{W}, 225 \mathrm{~m}$ ), macho, 13-28.VI.2000, R. Henriquez col., armadilha Malaise (IAHC).

\section{Cerambycini \\ Jupoata rufipennis (Gory, 1831)}

Cerambyx rufipennis Gory in Guérin-Méneville, 1831: 226, est. 44, figs. 1,1 a.

Brasilianus rufipennis; Martins, 1979: 28; Monné, 1993b: 6 (cat.). Jupoata rufipennis; Martins, 2002: 207.

Jupoata rufipennis tem vasta distribuição desde o Panamá até o Uruguai (MARTins 2002: 210). Para a Colômbia, foi assinalada para Muzo, Boyacá.
Material examinado. COLÔMBIA. Amazonas: PNN (Parque Nacional Natural) Amacayacu Matamata ( $\left.3^{\circ} 23^{\prime} \mathrm{S}, 70^{\circ} 06^{\prime} \mathrm{W}, 150 \mathrm{~m}\right), 2$ fêmeas, 27.III-03.IV.2000, A. Parente col., armadilha Malaise (IAHC, MZSP).

\section{Poeciloxestia carlyslei Fragoso, 1978}

Poeciloxestia carlyslei Fragoso, 1978: 134, figs. 166-170, 181-187; Monné, 1993b: 19 (cat.); Julio et. al., 2000: 25 (tipo).

Poeciloxestia carlyslei só era conhecida pelo material-tipo procedente da Colômbia, Cundinamarca: Monteredondo e Valle del Cauca: Cali.

Material examinado. COLÔMBIA. Boyacá: Miraflores (Vereda el Cajón, $5^{\circ} 11^{\prime} 50 " \mathrm{~N}, 7^{\circ} 08^{\prime} 56^{\prime \prime} \mathrm{W}, 1720$ m), fêmea, 24.III.1997, Miller $\&$ Mora col. (IAHC).

Poeciloxestia paraensis Lane, 1965

Poeciloxestia paraensis Lane, 1965: 274; Monné, 1993b:21 (cat.); Julio et. al., 2000: 25 (tipo)

Fragoso (1978) registrou a distribuição para Trinidad y Tobago, Venezuela, Guiana Francesa e Brasil (Pará).

Material examinado. COLÔMBIA. Cundinamarca: La Mesa (4³8’05"N, 74²7’57"W, 1298 m), fêmea, 18.VI.1997, E. Barón col. (IAHC).

\section{Elaphidionini \\ Ambonus electus (Gahan \& Arrow, 1903)}

Trichophorus electus Gahan \& Arrow, 1903: 254, est. 8, fig. 8. Ambonus electus; Duffy, 1953: 187; Monné, 1993c: 66 (cat.).

Registrada por MonNÉ(1993c) para Rondônia, Brasil central, nordeste do Brasil, Paraguai, Bolívia e Argentina. Chemsak et al. (1980) assinalaram-na para Honduras, uma distribuição disjunta. O material ora examinado, embora com localidade duvidosa, certamente é o primeiro registro para a Colômbia.

Material examinado. COLÔMBIA. Cundinamarca ou Boyacá ?, fêmea, 1992, Corradine col. (GMIC).

\section{Paramallocera cribripennis (Bates, 1885)}

Eurysthea cribripennis Bates, 1885: 256, est. 18, fig.5.

Paramallocera cribripennis; Aurivillius, 1912: 100; Monné, 1993c: 75 (cat.).

Paramallocera cribripennis foi assinalada para a Costa Rica, Panamá e Colômbia (Cundinamarca) por FonSECA-GESSNER (1990). Martínez (2000) registrou-a para Antioquia e Magdalena.

Material examinado. COLÔMBIA. Cundinamarca: Bogotá (Colégio Emílio Valenzuela), fêmea, 2.V.1987, Corradine col. (GMIC); Tabio (455'07"N, 7406'07"W, 2589 m), fêmea, 12.X.1997, M. Rico col. (IAHC). Boyacá: Moniquira $\left(5^{\circ} 52^{\prime} 51^{\prime \prime N}, 73^{\circ} 34^{\prime} 45^{\prime \prime} \mathrm{W}, 1650 \mathrm{~m}\right)$, macho, 12.III.1992, Luengas \& Medina col. (IAHC). 

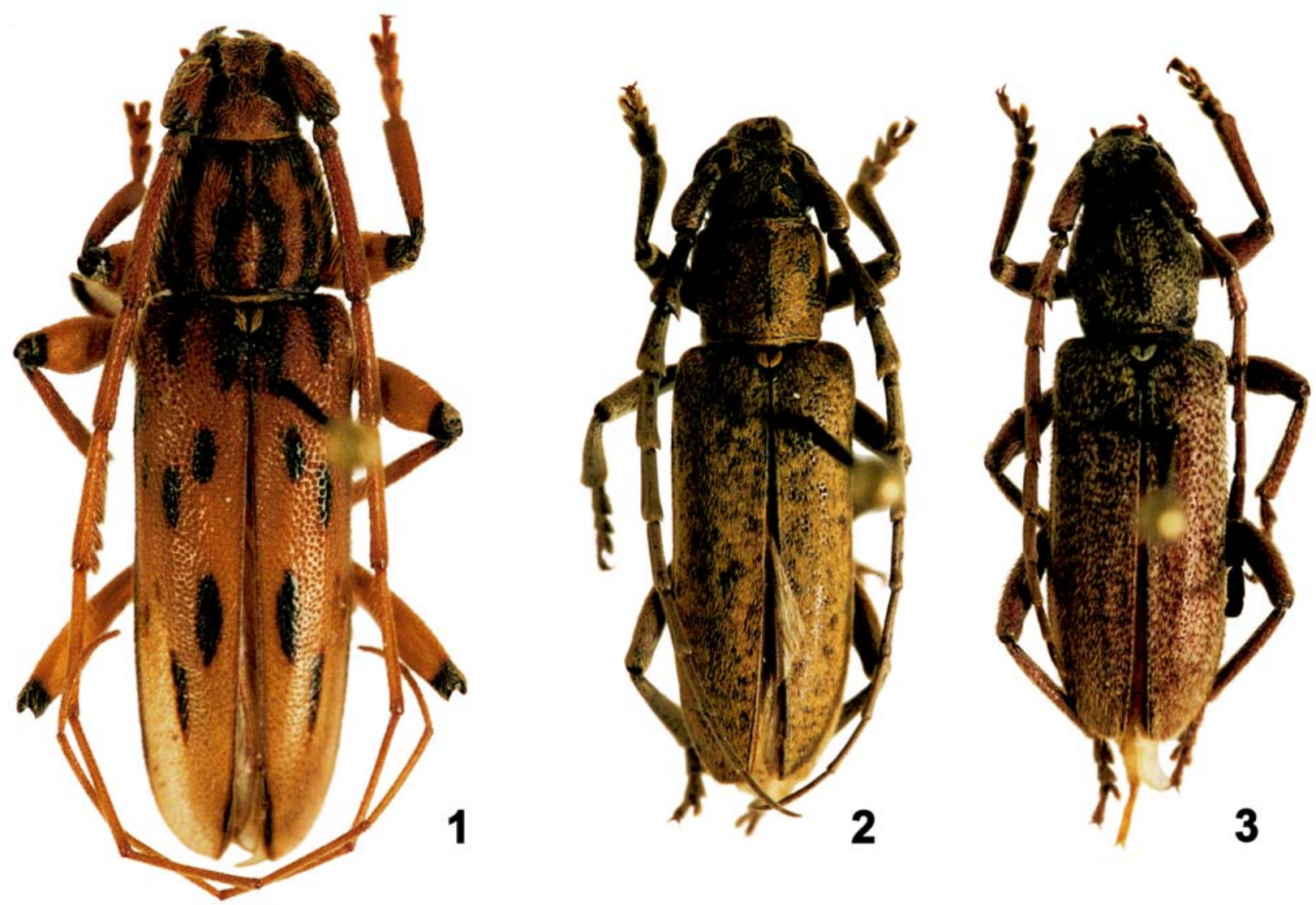

Figs. 1-3. 1, Achryson lineolatum Erichson, 1847, macho, de Macaravita, Santander, comprimento 18,5 mm; 2, Anelaphus colombianus sp. nov., holótipo macho, comprimento 16,2 $\mathrm{mm} ; \mathbf{3}$, Anelaphus sparsus sp. nov., holótipo fêmea, $14,1 \mathrm{~mm}$.

\section{Periboeum pubescens (Olivier, 1790)}

Cerambyx pubescens Olivier, 1790: 309.

Stenocorus pubescens; Fabricius, 1792: 301

Sphaerion pubescens; White, 1853: 108.

Periboeum pubescens; Lacordaire, 1869: 319; Monné, 1993c: 83 (cat.). Periboeum ebeninum Bates, 1870: 274.

Espécie tipicamente amazônica, foi assinalada por MonNÉ (1993c) para Trinidad y Tobago, Venezuela, Peru, Brasil (Amazônia) e Guianas (Guiana, Suriname e Guiana Francesa).

Material examinado. COLÔMBIA. Amazonas: PNN (Parque Nacional Natural) Amacayacu Matamata ( $\left.3^{\circ} 23^{\prime} \mathrm{S}, 70^{\circ} 06^{\prime} \mathrm{W}, 150 \mathrm{~m}\right)$, macho, 3-9.IV.2000, A. Parente col., armadilha Malaise (IAHC); macho, 7-14.VIII.2000, A. Parente col., armadilha Malaise (IAHC).

\section{Stizocera plicicollis (Germar, 1824)}

Cerambyx (Stenocorus) plicicollis Germar, 1824: 506.

Elaphidion plicicollis; Germar in Guérin-Méneville, 1839: 330.

Sphaerion plicicolle; White, 1853: 107.

Periboeum plicicollis; Lacordaire, 1869: 319.

Stizocera plicicollis; Gounelle, 1907: 242; Monné, 1993c: 9 (cat.);

Lingafelter, 1998: 90, fig. 132; Martínez, 2000: 87.

Esta espécie ocorre do México à Argentina. MARTínez (2000) assinalou-a para César, Cundinamarca e Meta na Colômbia.
Material examinado. COLÔMBIA. Córdoba: San Andrés (45'55"N, 75³7’48"W, 110 m), fêmea, 20.XI.1998, L. Banda col. (IAHC).

\section{Stizocera poeyi (Guérin-Méneville, 1838)}

Elaphidion poeyi Guérin-Méneville, 1838: 284.

Nephalius poeyi; Chevrolat, 1862: 268.

Periboeum poeyi; Thomson, 1878: 19.

Stizocera (Periboeum) poeyi; Bates, 1892: 152.

Stizocera poeyi; Gounelle, 1907: 242; Lingafelter, 1998: 93, fig. 134. Nesostizocera poeyi; Martins \& Napp, 1983: 88; Monné, 1993c: 5 (cat.).

Espécie largamente distribuída e ocorrente em Cuba, Panamá, Colômbia, Venezuela, Brasil e Bolívia (GiLmour 1968:136; MonNÉ 1993c). Gounelle (1909a:644) citou-a para a Colômbia e MARTínez (2000) para Antioquia.

Material examinado. COLÔMBIA. Tolima: Saldaña ( $3^{\circ} 56^{\prime} 05^{\prime \prime N}$, $\left.75^{\circ} 01^{\prime} 13 " \mathrm{~W}, 310 \mathrm{~m}\right)$, macho, 2.VII.1995, V. Capiño col. "trampa de luz" (IAHC).

\section{Pantonyssus glabricollis Fuchs, 1961}

Pantonyssus glabricollis Fuchs 1961: 7; Monné, 1993c: 80 (cat.).

Originalmente descrita do Brasil (Pará: Santarém), esta espécie parece ocorrer em toda Floresta Amazônica e registra- 
se agora para localidade bem a oeste da localidade-tipo, mas inserida na mesma formação vegetal. Com base em material do MCNZ, aproveitamos o ensejo para registrar a espécie para Roraima, Brasil.

Material examinado. COLÔMBIA. Vichada: PNN (Parque Nacional Natural) Tuparro $\left(5^{\circ} 21^{\prime} \mathrm{N}, 67^{\circ} 51^{\prime} \mathrm{W}, 100 \mathrm{~m}\right)$, macho, fêmea, 29.VII8.VIII.2000, M. Villalba col., "malaise" (IAHC, MZSP). BRASIL. Roraima: Rio Uraricoara (Ilha de Maracá), fêmea, 19-24.VII.1987, J. A. Rafael \& L. S. Aquino col., armadilha de Malaise (MCNZ).

\section{Pantonyssus suturale sp. nov.}

Fêmea. Cabeça e escapo pretos; pedicelo e flagelômeros, protórax, face ventral, fêmures e espinhos apicais, tíbias e tarsos amarelo-alaranjados. Élitros alaranjados, enegrecidos nos frisos: sutural, do meio à extremidade e marginal, do terço apical ao ápice (espinho apical externo inclusive). Dorso da cabeça esparsamente pontuado. Lobos oculares superiores com cinco fileiras de omatídios, tão distantes entre si quanto o dobro da largura de um lobo. Antenas atingem o ápice dos élitros na extremidade do antenômero IX. Escapo aplanado no dorso. Antenômeros III-VII com espinhos apicais. Pronoto com cinco gibosidades pouco notáveis; entre as gibosidades laterais e a central com rugas rasas. Élitros densamente pontuados no terço basal e sem pontos na metade apical. Tubérculo mesosternal manifesto.

Dimensões, em mm, fêmea. Comprimento total, 13,6; protórax: comprimento, 2,7, maior largura, 2,3; comprimento do élitro, 10,1; largura umeral, 3,1.

Material-tipo. Holótipo fêmea, COLÔMBIA, Santander: Macaravita (Hacienda El Cairo), 29.III.1994, Corradine col., à luz (GMIC).

Discussão. Espécies de Pantonyssus separam-se principalmente pela cor do corpo e dos apêndices. P. suturale sp. nov. distingue-se de todas as outras espécies pela coloração dos élitros com a metade apical dos frisos sutural e marginal enegrecidas. Na maioria das espécies, todo o quinto apical dos élitros é preto. $O$ pronoto com rugosidades entre as gibosidades é mais semelhante ao de $P$. erichsoni White, 1853 e P. erichsoni var. flavipes Fisher, 1944. Em P. erichsoni as antenas, pernas e pontas dos élitros são pretas e na variedade flavipes (talvez boa espécie), descrita da Venezuela, embora as pernas sejam alaranjadas, as pontas do dos élitros são pretas.

\section{Anelaphus colombianus sp. nov.}

(Fig. 2)

Macho.Tegumento castanho-escuro mais avermelhado nas pernas. Cabeça com pubescência amarelada esparsa, concentrada nos lados da fronte e ao redor dos olhos. Lobos oculares superiores com quatro fileiras de omatídios, tão distantes entre si quanto o dobro da largura de um lobo. Antenas atingem o ápice dos élitros. Antenômeros com depressão longitudinal no lado externo. Antenômeros III-VI com espinho interno; VII-X com espículo interno. Protórax mais largo que longo. Pronoto com pubescência densa amarelada; uma faixa estreita, longitudinal, brilhante, da constrição anterior à basal; lados dessa faixa com área indefinida revestida por pubescência muito esparsa que permite visualizar pontos $(32 \mathrm{x})$ grandes e próximos. Escutelo densamente pubescente com estreita faixa central, glabra. Élitros com pubescência amarelada densa com alguns espaços irregulares, pequenos, sem pubescência; setas escassas e mais curtas que o pedicelo. Extremidades elitrais transversalmente truncadas. Cavidades procoxais abertas atrás. Face ventral com pubescência amarelada, exceto no centro do metasterno. Lados do metasterno com pontuação moderada. Pernas revestidas por pubescência amarelada, moderada.

Fêmea. Colorido geral avermelhado. Lobos oculares superiores com distância entre si pouco maior que a largura de um lobo. Antenas tão longas quanto o corpo; com pubescência esparsa. Com relação aos machos, áreas deprimidas dos antenômeros menos profundas; pubescência dos élitros entremeadas por áreas glabras pouco maiores e pubescência da face ventral mais esparsa.

Dimensões, em mm, macho/fêmea. Comprimento total, 16,2/ 11,3; protórax: comprimento, 3,2/2,2, maior largura, 3,6/2,4; comprimento do élitro, 11,7/8,2; largura umeral 4,6/3,0.

Material-tipo. Holótipo macho, COLÔMBIA, Santander: Macaravita (Hacienda El Cairo), 27. XI.1989, Corradine col. (GMIC); parátipo fêmea, mesma localidade do holótipo, XII.1992, Corradine col. (MZSP).

Discussão. Anelaphus colombianus sp.nov. é semelhante a A. subseriatus (Bates, 1885) da qual examinamos exemplar de Cundinamarca: Fusagasugá (MCNZ). Difere pelas antenas tão longas quanto o corpo; pelo pronoto densamente pubescente com estreita faixa glabra central desnuda e pontuação pouco visível apenas nas áreas glabras laterais e pelas setas elitrais muito curtas. Em A. subseriatus (fêmea) as antenas atingem o quarto apical dos élitros; o pronoto é esparsamente pubescente (permite ver a pontuação); a área glabra do pronoto é mais larga, mais curta e atinge o meio do pronoto; os pêlos dos élitros, principalmente próximo ao ápice, são mais longos que o pedicelo.

\section{Anelaphus sparsus sp. nov.}

(Fig. 3)

Fêmea. Colorido geral avermelhado ou vermelho acastanhado. Fronte mais longa do que larga com pubescência esbranquiçada. Occipício glabro. Lobos oculares superiores com quatro fileiras de omatídios, tão distantes entre si quanto a largura de um lobo. Antenas atingem o quinto apical dos élitros. Antenômeros com áreas deprimidas pouco demarcadas. Antenômeros III-VI com espinho apical interno; VII com espículo diminuto. Pronoto com pubescência esbranquiçada pouco densa, com três áreas glabras, pequenas: duas láteroanteriores e uma centro-posterior; pontuação aparente no disco. Escutelo revestido por pubescência esbranquiçada. Élitros com pubescência esbranquiçada uniforme; setas muito abundantes 
em toda a superfície. Extremidades elitrais arredondadas. Cavidades procoxais fechadas atrás. Face ventral com pubescência esbranquiçada (menos em estreita área centroposterior do metasterno), entremeada por setas especialmente nos urosternitos.

Dimensões, em mm, fêmea. Comprimento total, 14,1-14,4; protórax: comprimento, 2,8-3,1 , maior largura, 3,3-3,3 ; comprimento do élitro, 9,9-10,0; largura umeral, 4,1-4,4.

Material-tipo. Holótipo fêmea, COLÔMBIA, Magdalena: PNN (Parque Nacional Natural) Tayrona Palangana $\left(11^{\circ} 20^{\prime} \mathrm{N}, 7^{\circ} 02^{\prime} \mathrm{W}, 30\right.$ m), 21.II-5.III.2001, R. Henriquez col. (IAHC); parátipo fêmea, mesmos dados do holótipo (MZSP).

Discussão. Anelaphus sparsus sp. nov. caracteriza-se pelas cavidades procoxais fechadas atrás, no que contraria a diagnose genérica apresentada por LiNGAFELTER (1998: 52). Entretanto, concorda com outros caracteres e parece-nos apropriado situá-la em Anelaphus. Além disso, distingue-se de A. colombianus sp. nov. pela pubescência corporal esbranquiçada; pelo pronoto com pubescência esparsa e áreas glabras; pelos élitros com pubescência mais esparsa; pelas setas elitrais muito mais abundantes e pelos ápices dos élitros arredondados.

\section{Piezocerini Haruspex lineolatus Bates, 1870}

Haruspex lineolatus Bates, 1870: 280; Monné, 1993d: 24 (cat.).

MARTINS (1976a) assinalou H. lineolatus para a Venezuela, Guiana, Guiana Francesa, Brasil (Amazonas, Pará), Peru, Equador e Bolívia.

Material examinado. COLÔMBIA. Amazonas: PNN (Parque Nacional Natural) Amacayacu Matamata ( $3^{\circ} 23^{\prime} \mathrm{S}, 70^{\circ} 06^{\prime} \mathrm{W}, 150 \mathrm{~m}$ ), fêmea, 12-19.III.2000, A. Parente col., armadilha Malaise (IAHC); fêmea, 20-26.III.2000, A. Parente col., armadilha Malaise (MZSP).

\section{Eligmodermini \\ Eligmoderma Thomson, 1864}

Eligmoderma Thomson, 1864: 246; Monné, 1993d: 1 (cat.).

Aurivilluus (1923:442) publicou chave para identificação das espécies de Eligmoderma na qual E. ziczac Nonfried, 1895 se distingue por apresentar élitros castanhos com apenas duas faixas amareladas em zigue-zague, protórax com lados desarmados e tão longo quanto largo. O exemplar colombiano que identificamos como E. ziczac é um macho que coincide perfeitamente com a descrição original e com os dados de chave reproduzidos acima.

No mesmo trabalho, Auriviluius (l. c.) publicou descrição e figura de E. spinicolle Aurivillius, 1923 procedente da Colômbia (sem localidade precisa). Julgou, com dúvida, que o holótipo fosse uma fêmea. Identificamos uma fêmea que coincide perfeitamente com a descrição e a figura.

Com exceção do protórax, os dois exemplares poderiam ser interpretados como ambos os sexos de uma mesma espécie.
No macho, que corresponde a $E$. ziczac, o protórax tem pequena gibosidade nos lados e na fêmea, que corresponde a $E$. spinicolle, os lados do protórax têm espinho central longo e tubérculo ao nível do terço anterior.

Como só dispomos de um casal, julgamos o material insuficiente para estabelecer uma sinonímia formal e registramos as duas espécies separadamente.

\section{Eligmoderma ziczac Nonfried, 1895}

Eligmoderma ziczac Nonfried, 1895: 310; Monné, 1993d: 2 (cat.).

Material examinado. COLÔMBIA. Casanare: Yopal $\left(5^{\circ} 21^{\prime} \mathrm{N}\right.$, 72²4'W, 350 m), fêmea, 14.V.1972, C. Baquero col. (IAHC).

Eligmoderma spinicolle Aurivillius, 1923

Eligmoderma spinicolle Aurivillius, 1923: 441; Monné, 1993d: 2 (cat.).

Material examinado. COLÔMBIA. Cundinamarca: Cachipay ( $\left.5^{\circ} 16^{\prime} 22^{\prime \prime} \mathrm{N}, 74^{\circ} 34^{\prime} 22^{\prime \prime} \mathrm{W}\right)$, macho, 2.IV.1972, A. Martínez col. (IAHC).

Agradecimento. A Antonio Santos Silva (MZSP) pela execução das fotografias.

\section{REFERÊNCIAS}

Aurivilius, C. 1912. Coleopterorum Catalogus, pars 39, Cerambycidae: Cerambycinae. Berlin, W. Junk, 574 p.

Aurivilius, C. 1923. Neue oder wenig bekannte Coleoptera Longicornia. 19. Arkiv für Zoology 15(25): 437-479, 21 figs.

Barriga T. J. E.; S. Tomislav-Curcovik; T. Fichet L.; J. L. Henríguez S. \& J. MaCAYA B. 1993. Nuevos antecedentes de coleopteros xilofagos y plantas hospederas en Chile, com una recompilación de citas prévias. Revista Chilena de Entomologia 20: 65-91.

Bates, H. W. 1870. Contributions to an insect fauna of the Amazon Valley (Coleoptera: Cerambycidae). Transactions of the Entomological Society of London 1870: 243-335.

Bates, H. W. 1879-1885. Biologia Centrali-Americana, Insecta, Coleoptera. Londres, British Museum (Natural History). v. 5, 436 p., 24 est.

Bates, H. W. 1892. Additions to the longicornia of Mexico and Central America, with remarks on some of the previously recorded species. Transactions of the Entomolological Society of London 1892: 143-183, 2 pls.

BRUCH, C. 1908. Longicórnios argentinos nuevos o poco conocidos. I. Revista del Museo de La Plata 15: 198-220.

Chemsak, J. A.; E. G. Linsley \& J. V. Mankins. 1980. Records of some Cerambycidae from Honduras. Pan-Pacific Entomologist 56(1): 26-37.

Chevrolat, L. A. A. 1862. Coléoptères de l'Ile de Cuba. Notes, synonymies et descriptions d'espèces nouvelles. Familles des cérambycides et des parandrides. Annales de la Societé Entomologique de France (4)2: 245-280.

Di IORIO, O. R. 1994. Cerambycidae (Coleoptera) y plantas hospedadoras del noreste de Argentina. Revista Brasileira de Entomologia 38(1): 15-22.

Di IoRio, O. R. 1997. Plantas hospedadoras y biogeografía de Cerambycidae (Coleoptera) del noroeste y centro de la Argentina. Revista de Biologia Tropical 45(1): 149-158.

Duffy, E. A. J. 1953. A monograph of the immature stages of Britsh and imported timber beetles (Cerambycidae). London, British Museum (Natural History), VIII+350 p.

Erichson, W. F. 1847. Conspectus insectorum coleopterorum quae in Republica Peruana observata sunt. Archiv für Naturgeschichte 13: $67-85$.

Fabricius, J. C. 1792. Entomologia Systematica... Hafniae. v. 1: $\mathrm{XX}+538 \mathrm{p}$. 
FIsHeR, W. S. 1944. Cerambycidae of Caripito, Venezuela. Zoologica 29: 3-12.

Fonseca-Gessner, A. A. 1990. Revisão taxonômica do gênero Paramallocera Aurivillius, 1912 (Coleoptera, Cerambycidae, Cerambycinae, Sphaerionini). Revista Brasileira de Entomologia 34(4): 817-856.

Fragoso, S. A. 1978. Revision of the neotropical genus Poeciloxestia Lane, 1965. Coleopterists Bulletin 32(2): 113-158.

Fuchs, E. 1961. 4. Beitrag zur Kenntnis der neotropischen Cerambyciden. Koleopterologische Rundschau 39: 6-21.

Gahan, C. J. \& G. J. Arrow. 1903. List of the Coleoptera collected by Mr. A. Robert at Chapada, Matto Grosso (Percy Sladen Expedition to Central Brazil). Procedings of the Zoological Society of London 2: $244-258,1 \mathrm{pl}$

Galileo, M. H. M. \& U. R. Martins. 2003. Cerambycidae (Coleoptera) da Colômbia. III. Cerambycinae com olhos finamente granulados. Iheringia, Série Zoologia 93(1): 31-36.

Germain, P. 1898. Apuntes entomolójicos. Los lonjicórnios chilenos. Anales de la Universidad de Chile 100: 541-562; 641-676; 781-815 (Separata, 274 p., 3 est.).

Germar, E. F. 1824. Insectorum species novae aut minus cognitae, descriptionibus illustratae. Halae. XXIV+624 p.

Germar, E. F. 1839. In: F. E. GuÉrin-Méneville. Note synonymique sur les cérambycins décrits par M. Germar, dans son Insectorum species novae aut minus cognitae descriptionibus illustrate, Halae, 1824. Revue de Zoologie 1839: 329-331.

Gory, H. L. In: F. E. Guérin-Méneville. 1831. Iconographie du Régne Animal de G. Cuvier (Insectes). Paris, Baillière. iv + 5-576, 110 pls.

Gounelle, E. 1907. Note sur les genres Sphaerion Serv., Nephalius Newm., Mephritus Pasc., Periboeum Thoms. et Stizocera Serv. Bulletin de la Societé Entomologique de France 1907: 238244.

Gounelle, E. 1909a. Liste des cérambycides de la région de Jatahy, Etat de Goyaz, Brésil. Annales de la Societé Entomologique de France 77: 587-688.

Gounelle, E. 1909b. Notes synonymiques (Col.). Bulletin de la Societé Entomologique de France 1909: 304-305.

Gilmour, E. F. 1968. The Coleoptera Cerambycidae of Curação, Aruba and Bonaire. Studies of the Fauna of Curaçao, Aruba and Bonaire 25(100): 83-178, 10 pls.

GuÉRIN-MÉNEviLle, F. E. 1838. Insectes coléoptères inédits, découverts par M. Lanier dans l'intérieur de l'Ile de Cuba. Revue de Zoologie 1838: $279-286$.

Joly T., L. J. 2000. Revisión de las especies venezolanas del género Achryson Audinet-Serville (Coleoptera: Cerambycidae: Achrysonini). Boletin de Entomologia Venezolana 15(2): 147-179.

Julio, C. E. de A.; J. A. Giorgi \& M. A. Monné. 2000. Os tipos primários de Cerambycidae (Coleoptera) da coleção do Museu Nacional - Rio de Janeiro. Publicações Avulsas do Museu Nacional (84): 1-54.

KIRSCH, T. F. 1889. Coleopteren gesammelt in den Jahren 1868-1877 auf einen Reise durch Süd Amerika von Alphons Stübel. Abhandlungen Zoologische Museum 4: 1-58.

Lacordaire, J. T. 1869. Genera des Coléoptères...v. 8. Paris, Librairie Encyclopédique de Roret, $552 \mathrm{p}$.

Lane, F. 1965. Cerambycoidea Neotropica nova VI. (Coleoptera). Studia Entomologica 8: $269-336$

LinelL, M. L. 1898. On the coleopterous insects of Galápagos Islands. Proceedings of the United States National Museum 21(1143): 249-268.

Lingafelter, S. W. 1998. The Genera of Elaphidiini Thomson 1864. Memoirs of the Entomological Society 20: 1-118.

Linsley, E. G. \& J. A. Chemsak. 1966. Cerambycidae of the Galápagos Islands. Proceedings of the California Academy of Sciences (4) 33(8): 197-236.

MarTíneZ, C. 2000. Escarabajos Longicórnios (Coleoptera: Cerambycidae) de Colômbia. Biota Colombiana 1(1): 76-105.

Martins, U. R. 1971. Notas sôbre Cerambycinae VI. Subsídios ao conhecimento dos Ibidionini. Arquivos de Zoologia 21(3): 121178.

Martins, U. R. 1973. Notas sôbre Cerambycinae VIII. Papéis Avulsos de Zoologia 26(1): 201-213.

Martins, U. R. 1976a. Sistemática e evolução da tribo Piezocerini. Arquivos de Zoologia 27(3-4): 165-370.

Martins, U. R. 1976b. Notas sôbre Achryson Serville, 1833. Revista Brasileira de Entomologia 20(2): 73-78.

Martins, U. R. 1979. Notas e descrições em Brasilianus Jakobson, 1924 (Coleoptera, Cerambycidae). Revista Brasileira de Entomologia 23(1): 23-28.

Martins, U. R. 1998. Cerambycidae Sul-Americanos (Coleoptera). São Paulo, Sociedade Brasileira de Entomologia v. 2, 195 p.

Martins, U. R. 2002. Cerambycidae Sul-Americanos (Coleoptera). São Paulo, Sociedade Brasileira de Entomologia v. 4, IV+265 p.

Martins, U. R. \& M. H. M. Galileo. 2002. Cerambycidae (Coleoptera) da Colômbia. I. Eburiini (Cerambycinae). Iheringia, Série Zoologia 92(4): $5-10$.

Martins, U. R. \& M. H. M. Galileo. 2002. Cerambycidae (Coleoptera) da Colômbia. II. Ibidionini (Cerambycinae). Iheringia, Série Zoologia 92(4): 11-18

Martins, U. R. \& D. S. Napp. 1983. Gêneros Stizocera Audinet-Serville, Nesostizocera Linsley e Parastizocera Linsley: chaves para espécies, descrições, notas e sinonímias. Papéis Avulsos de Zoologia 35(7): 79-92.

Monné, M. A. 1972. Revisión parcial del género Achryson Serville, 1833 (Coleoptera, Cerambycidae, Achrysonini). Revista Peruana de Entomologia 15: 103-112.

Monné, M. A.1993a. Catalogue of the Cerambycidae (Coleoptera) of the western hemisphere, Part I. Subfamily Cerambycinae: Tribes Erlandiini, Smodicini, Oemini, Methiini, Xystrocerini, Dodecosini, Opsimini, Achrysonini and Pleiarthrocerini. São Paulo, Sociedade Brasileira de Entomologia, II+76 p.

Monné, M. A. 1993b. Catalogue of the Cerambycidae (Coleoptera) of the western hemisphere. Part III. Subfamily Cerambycinae: Tribes Cerambycini, Diorini and Piezocerini. São Paulo, Sociedade Brasileira de Entomologia, $52 \mathrm{p}$.

MonnÉ, M. A. 1993c. Catalogue of the Cerambycidae (Coleoptera) of the western hemisphere. Part IV. Subfamily Cerambycinae: Tribe Elaphidionini. São Paulo, Sociedade Brasileira de Entomologia, $129 \mathrm{p}$.

Monné, M. A.1993d. Catalogue of the Cerambycidae (Coleoptera) of the western hemisphere. Part VI. Tribes Eligmodermini, Callidiopini, Curiini, Gracilliini, Obriini, Eumichtini, Phlyctaenodini, Holopterini, Stenoderini, Pseudocephalini and Bimiini. São Paulo, Sociedade Brasileira de Entomologia, II +47 p.

NAPP, D. S. \& Martins, U. R. 1982. Subsídios para uma revisão da tribo Achrysonini (Coleoptera, Cerambycidae) nas Américas. Papéis Avulsos de Zoologia 34: 349-401.

Nonfried, A. F. 1895. Coleoptera nova exotica. Berliner Entomologische Zeitschrift 40:279-312.

Olivier, A. G. 1790. Encyclopédie Methodique. Histoire Naturelle, Insectes. v. 5, $368 \mathrm{p}$.

Perroud, B. P. 1855. Description de quelques espèces nouvelles ou peu connus et création de quelques nouveaux genres dans lafamille des longicornes. Annales de la Societé Linneene de Lyon (2)2: $327-401$

Schoenherr, C. J. 1817. Synonymia Insectorum, oder: Versuch einer Synonymia aller bisher bekannten Insecten; nach Fabricii Systema Eleutheratorum \&c. geordnet. Skara, Lewerentzischen Buchdruekerey, v.1(3), xi $+506 \mathrm{p}$.

Thomson, J. 1864. Systema cerambycidarum ou exposé de tous les genres compris dans la famille des cérambycides et familles limitrophes. Mémoires de la Societé Royal des Sciences 19: 1-540.

Thomson, J. 1878. Typi cerambycidarum Musei Thomsoniani. E. Deyrolle, $21 \mathrm{p}$.

Tippmann, F. F. 1960. Studien über neotropische Longicornier III. Koleopterologische Rundschau 37-38: 82-217.

Villiers, A. 1980. Coléoptères Cerambycidae des Antilles francaises, II. Cerambycinae. Annales de la Societé Entomologique de France (N. S.) 16(2): 265-306

White, A. 1853. Catalogue of the coleopterous insects in the collection of the British Museum. Logicornia 1. Londres, British Museum (Natural History), v. 7: 1-174, est. 1-4. 